\title{
ION BEAM CHANNELING AND HYPERFINE INTERACTION ANALYSIS FOR THE CHARACTERIZATION OF STOICHIOMETRY AND ANTI-SITE POPULATION IN $\mathrm{LiNbO}_{3}$
}

\author{
A. Kling ${ }^{1)}$, L. Rebouta ${ }^{2)}$, J.G. Marques ${ }^{1)}$, J.G. Correia ${ }^{1,3)}$, M.F. da Silva ${ }^{4}$, \\ E. Diéguez ${ }^{5)}$, F. Agulló-López ${ }^{5)}$, J.C. Soares ${ }^{1)}$ \\ and the ISOLDE Collaboration, CERN
}

\begin{abstract}
Combined high precision RBS/channeling, NRA/channeling and perturbed angular studies were used to characterize near-stoichiometric and congruent $\mathrm{LiNbO}_{3}$ single crystals. In congruent $\mathrm{LiNbO}_{3}$ two non-equivalent $\mathrm{Li}$ sites are distinguished whereas in near-stoichiometric $\mathrm{LiNbO}_{3}$ only one of them exists. A comparison of the experimental results with Monte Carlo simulations was performed to check the validity of the different models for the structure of congruent lithium niobate crystals.
\end{abstract}

$$
\text { ( IS01 - 13) }
$$

Presented at the 12th Int. Conf. on Ion Beam Analysis, Tucson, USA (1995) Accepted for publication in Nucl. Instrum. Methods B

\footnotetext{
1) CFN, Univ. Lisboa, Av. Prof. Gama Pinto 2, P-1699 Lisboa, Portugal

2) Dep. Física, Univ. Minho, 4719 Braga, Portugal

3) PPE Div., CERN, CH-1211 Geneva 23, Switzerland

4) Dep. Física, ITN, E.N.10, P-2685 Sacavém, Portugal

5) Dep. Física de Materiales, Univ. Autonoma, 28049 Madrid, Spain
} 


\title{
Ion Beam Channeling and Hyperfine Interaction Analysis for the characterization of stoichiometry and anti-site population in $\mathrm{LiNbO}_{3}$
}

\author{
A. Kling ${ }^{1}$, L. Rebouta ${ }^{2}$, J.G. Marques ${ }^{1}$, J.G. Correia ${ }^{1,3}$, M.F. da Silva ${ }^{4}$, \\ E. Dieguez ${ }^{5}$, F. Agulló-López ${ }^{5}$, J.C. Soares ${ }^{1}$ and ISOLDE Collaboration, CERN \\ ${ }^{1}$ Centro de Física Nuclear da Universidade de Lisboa, Av. Prof. Gama Pinto 2, \\ 1699 Lisboa Codex, Portugal \\ ${ }^{2}$ Dep. Física, Universidade do Minho, 4719 Braga, Portugal \\ ${ }^{3}$ PPE Div., CERN, CH-1211 Geneva 23, Switzerland \\ ${ }^{4}$ Dep. Física, ITN, 2685 Sacavém, Portugal \\ ${ }^{5}$ Dep. Física de Materiales, C-IV, Universidad Autonoma, 28049 Madrid, Spain
}

\section{Introduction}

Lithium niobate $\left(\mathrm{LiNbO}_{3}\right)$ is an important material for applications in optoelectronic and integrated optics devices. Many of its properties are influenced by intrinsic defects due to deviations from the stoichiometry and extrinsic defects introduced by dopants. Crystals grown by the Czochralski technique from a congruently melting composition have a $\mathrm{Li}$ to $\mathrm{Nb}$ ratio $[\mathrm{Li}] /[\mathrm{Nb}]=0.945$, which differs from the 1:1 ratio inferred from the chemical formula $\mathrm{LiNbO}_{3}$. The $\mathrm{Li}$ deficiency leads to a defect structure for which several models have been proposed. According to the most common model by Abrahams and Marsh [1], based on Xray diffraction measurements, $\mathrm{Nb}$ occupies about $5.9 \%$ of the $\mathrm{Li}$ sites forming niobium antisites $\left(\mathrm{Nb}_{\mathrm{Li}}\right)$. In order to obtain charge compensation, $4.8 \%$ of the $\mathrm{Nb}$ sites have to be vacant. In contrast, Lerner et al. [2] suggested that the $\mathrm{Nb}$ lattice may not contain any vacancies and the number of $\mathrm{Nb}$ antisites is limited to the excess $\mathrm{Nb}$ fraction of $1.2 \%$. Charge compensation would than be achieved by a sufficient number of Li vacancies. An alternative model, first proposed by Nassau and Lines [3], explains the defect structure of congruent $\mathrm{LiNbO}_{3}$ by the existence of stacking fault type defects. Recent computational results obtained by Donnerberg et al. [4] indicate that these defects could be ilmenite-type stacking sequences probably forming substantial intergrowths. 
The recent characterization of bulk stoichiometric $\mathrm{LiNbO}_{3}$ crystals grown from melts containing $6 \% \mathrm{~K}_{2} \mathrm{O}$ [5] showed a significant change of some properties, such as decrease in the linewidths of ${ }^{93} \mathrm{Nb} \mathrm{NMR}$, in the $\mathrm{Fe}^{3+}$ electron paramagnetic resonance and in the Raman line of the $\mathrm{E}$ phonon at $153 \mathrm{~cm}^{-1}$ [5], but no correlation to the defect structure of congruent $\mathrm{LiNbO}_{3}$ was made.

Ion beam channeling and hyperfine interaction measurements are extremely sensitive to structural properties of single crystals and yield complementary information [6]. Using these techniques, a comparative study of the defect properties of both near-stoichiometric and congruent lithium niobate crystals is presented in this work.

\section{Experimental details}

Near-stoichiometric $\mathrm{LiNbO}_{3}$ crystals were grown at the Universidad Autonoma of Madrid using the Czochralski method from a melt containing grade I JohnsonMathey powder to which $4.6 \% \mathrm{~K}_{2} \mathrm{O}$ were added. Congruent $\mathrm{LiNbO}_{3}$ crystals, also grown by the Czochralski method, were obtained from Munich and Budapest. Samples were cut both parallel and perpendicular to the c-axis and polished. PIXE analysis of the near-stoichiometric plates indicates that potassium is not incorporated in the crystals down to the detection level of $100 \mathrm{ppm}$. The optical absorption measurements show an optical absorption edge $\left(\alpha=20 \mathrm{~cm}^{-1}\right)$ at $314 \mathrm{~nm}$ for the near-stoichiometric crystal, and at $320 \mathrm{~nm}$ for a congruent one. From the calibration curve given in Ref. 7 a $\mathrm{Li}$ to $\mathrm{Nb}$ ratio of $[\mathrm{Li}] /[\mathrm{Nb}]=0.979$ for the near stoichiometric crystal was derived.

The RBS/C and NRA/C experiments were carried out at the $3.1 \mathrm{MeV}$ Van de Graaff accelerator at Sacavém. The orientation of the crystals was checked using a 1.6 $\mathrm{MeV} \mathrm{He}^{+}$beam. The channeling investigations of the Nb-RBS and the Li-NRA with the ${ }^{7} \mathrm{Li}(\mathrm{p}, \alpha)^{4} \mathrm{He}$ reaction were performed using both 1.5 and $1.6 \mathrm{MeV} \mathrm{H}^{+}$ beams. The $\alpha$ particles with an energy of about $7.5 \mathrm{MeV}$ released in this reaction can be easily distinguished from the backscattered protons. The charged particles were detected at an angle near $180^{\circ}$ using an annular silicon surface barrier detector with a resolution of $18 \mathrm{keV}$. 
For the hyperfine interaction measurements, radioactive ${ }^{111 \mathrm{~m}} \mathrm{Cd}$ was implanted at $60 \mathrm{keV}$ energy using the ISOLDE/CERN isotope separator, to doses of $5 \times 10^{12}$ $\mathrm{at} / \mathrm{cm}^{2}$. The projected range of the $\mathrm{Cd}^{+}$ions is $23 \mathrm{~nm}$ and the peak concentration is $1 \times 10^{18} \mathrm{Cd} / \mathrm{cm}^{3}$, i.e., about 0.02 at $\%$. After implantation the samples were annealed in air at $873 \mathrm{~K}$ for $30 \mathrm{~min}$. and slowly cooled down to the measuring temperature of $295 \mathrm{~K}$. The $\mathrm{e}^{-}-\gamma$ PAC experiments were performed using the well-known 151$245 \mathrm{keV}$ cascade from the decay of ${ }^{111 \mathrm{~m}} \mathrm{Cd}$ with an anisotropy $\mathrm{b}_{2} \mathrm{~A}_{22}=+0.230$. The $245 \mathrm{keV}$ state has $\operatorname{spin} \mathrm{I}=5 / 2$, half-life of $\mathrm{T}_{1 / 2}=84 \mathrm{~ns}$ and quadrupole moment of $|\mathrm{Q}|=0.81(13) \mathrm{b}[8]$. The experimental set-up consists of two $\beta$ spectrometers of the Siegbahn type for the detection of conversion electrons and two $\mathrm{BaF}_{2}$ scintillators for $\gamma$ detection, arranged in a plane at $90^{\circ}$ intervals [9], with a time resolution of 1.0 ns $(\mathrm{FWHM})$. From the coincidence spectra $\mathrm{N}(\theta, \mathrm{t})$, where $\theta$ is the angle between detectors and $\mathrm{t}$ is the time delay between events, the time differential anisotropy $\mathrm{R}(\mathrm{t})=2 \cdot\left[\mathrm{N}\left(180^{\circ}, \mathrm{t}\right)-\mathrm{N}\left(90^{\circ}, \mathrm{t}\right)\right] /\left[\mathrm{N}\left(180^{\circ}, \mathrm{t}\right)+2 \cdot \mathrm{N}\left(90^{\circ}, \mathrm{t}\right)\right] \sim \mathrm{b}_{2} \mathrm{~A}_{22} \mathrm{G}_{22}(\mathrm{t})$ was calculated . $\mathrm{G}_{22}(\mathrm{t})$ is the perturbation function which describes the modulation of the angular correlation. For a cascade with $I=5 / 2$ three frequencies $\omega_{n}=C_{n}(\eta) v_{Q}$ are observed, giving

$$
G_{22}(t)=\sum_{n=0}^{3} S_{2 n} \cos \left(C_{n}(\eta) v_{Q} t\right) \exp \left(-\frac{1}{2} \delta C_{n}(\eta) v_{Q} t\right)
$$

The quadrupole coupling constant $\mathrm{v}_{\mathrm{Q}}=\mathrm{eQV} \mathrm{Zz} / \mathrm{h}$ and the asymmetry parameter $\eta=$ $\left(\mathrm{V}_{\mathrm{xx}}-\mathrm{V}_{\mathrm{yy}}\right) / \mathrm{V}_{\mathrm{zz}}$, which can be deduced from the frequency factors $\mathrm{C}_{\mathrm{n}}(\eta)$, contain information about the magnitude of the principal component $\mathrm{V}_{\mathrm{zz}}$ and the asymmetry of the EFG. The exponential factor allows for a Lorentzian distribution of EFGs around a mean value. Such a distribution can be caused e.g. by different lattice defects in the vicinity of the probe atoms. The $S_{2 n}$ coefficients, which give the amplitudes for each frequency, can be calculated for polycrystalline as well as for single crystalline samples. If fractions $f_{i}$ of the total number of radioactive probes are in different but unique sites experiencing different EFGs the observed spectra is described by a sum over several perturbation functions, $f_{i} \cdot G_{22}{ }^{i}(t)$, with the sum of the $f_{i}$ normalized to unity. 


\section{Results and Discussion}

\subsection{RBS/C and NRA/C}

Figs.1a) and b) show NRA spectra of the ${ }^{7} \mathrm{Li}(\mathrm{p}, \alpha)^{4} \mathrm{He}$ reaction for $1.5 \mathrm{MeV}$ protons aligned with the $\langle 11 \overline{2} 0\rangle$ axis and in random direction, for congruent and stoichiometric $\mathrm{LiNbO}_{3}$, respectively. The minimum yield derived from the aligned spectrum, near the surface $(1.5 \mu \mathrm{m})$, is remarkably lower for the stoichiometric crystal, $\chi_{\min }=53 \%$, than for the congruent samples: $\chi_{\min }=74 \%$ (Budapest) and $\chi_{\min }=73 \%$ (Munich, not shown here). From recent experiments on the dependence of the Li-NRA minimum yield on the Mg concentration [10] it is known that even for high $\mathrm{Mg}$ concentrations no $\chi_{\min }$ lower than $60 \%$ is observed for this axis. Both observations show that the Li sublattice in the case of near-stoichiometric $\mathrm{LiNbO}_{3}$ must be remarkably closer to the perfect arrangement.

Additionally, the aligned spectrum for the stoichiometric sample exhibits a much higher yield than the random spectrum for $\alpha$-particles emerging from higher depths. This can be attributed to a significantly lower stopping power in the axial direction for the stoichiometric crystal, which is expected for channeling in a perfect lattice. The resulting higher proton energy at a given depth and the

corresponding higher nuclear cross section for the ${ }^{7} \mathrm{Li}(\mathrm{p}, \alpha)^{4} \mathrm{He}$ reaction leads to an enhanced yield.

Figs.2a) and b) show $\mathrm{Li}$ and $\mathrm{Nb}$ angular scans for the (0001) plane and the $<11 \overline{2} 0>$ axis along the (0001) plane, respectively. For planar channeling, the LiNRA peaks observed for the stoichiometric and congruent crystals differ significantly in width and height. This hints to substantial differences in the structure of the Li sublattices. Also the Li-NRA angular scan for the $\langle 11 \overline{2} 0\rangle$ axis along the (0001) plane corroborates the differences between both crystals.

Computer simulations using the CASSIS code [11] show that in the case of stoichiometric lithium niobate the (0001) angular scan can be described assuming a perfect crystal lattice, as shown in Fig. 3. For the congruent crystals, simulation results obtained assuming different defect models previously discussed [1-4] are depicted in Fig.4. The experimental data are also included for comparison. 
The models, that correlate the defect structure only to the niobium antisites, just yield a decrease of the Li-NRA peak height (Fig. 4a and 4b), but do not reproduce the experimentally observed broadening. Only the assumption of two different lithium sites as derived from the occurrence of cation stacking faults with inversion symmetry (ilmenite type) results in both a decrease and broadening of the Li-NRA peak (Fig. 4c). In this case assuming a fraction of $2 \%$ dilute randomly distributed stacking faults yields a better fit to the experimental data is obtained.

The angular scans measured for Nb-RBS almost overlap for both congruent and stoichiometric samples. For all defect models only a minor influence on the $\mathrm{Nb}$ angular scans is expected, since a small fraction of $\mathrm{Nb}$ in $\mathrm{Li}$ sites (either $1.2 \%$ or $5.9 \%$ ) would only yield a small increase in the Nb-RBS minimum yield. In the case of the stacking fault model the additional $\mathrm{Nb}$ site in the c-plane is projected close to the regular one and therefore cannot be easily distinguished by RBS.

\subsection{Perturbed Angular Correlation}

Figs. 5a) and b) show the PAC spectra and respective Fourier analysis obtained for the congruent and the stoichiometric sample, respectively. The c-axis of the single crystals was in the detectors plane at $45^{\circ}$ with two detectors.

The amplitude of the time differential anisotropy $R(t)$ at $t \sim 200 n s$ is clearly larger for the stoichiometric crystal than for the congruent one. As discussed previously [12] for measurements in a congruent crystal, ${ }^{111} \mathrm{Cd}$ replaces $\mathrm{Li}$ but the PAC spectra cannot be described by only one quadrupole interaction frequency. In fact, the Fourier analysis of the spectra shows a second frequency with a value only slightly higher than the main component. The best fit to the data was obtained considering two nearby Li-site frequencies, $v_{Q}{ }^{I}=191(2) \mathrm{MHz}$ and $v_{Q}{ }^{I I}=205(2) \mathrm{MHz}$, with relative site occupancies of $71(5) \%$ and $29(5) \%$, respectively. Due to the symmetry properties of the $\mathrm{LiNbO}_{3}$ lattice the EFG expected at the $\mathrm{Li}$ site should have axial symmetry (i.e., $\eta=0$ ). However, the spectra can only be described assuming $\eta>0$ for both sites, $\eta^{\mathrm{I}}=0.11(2)$ and $\eta^{\mathrm{II}}=0.16(4)$. In contrast, the Fourier analysis of the spectra taken for the stoichiometric crystal shows sharper lines and the data are well described by one frequency only, $v_{\mathrm{Q}}=191(2) \mathrm{MHz}$, and an axially 
symmetric EFG, i.e., $\eta=0$. This indicates a higher lattice perfection for the stoichiometric sample. A study of the stoichiometric $\mathrm{LiNbO}_{3}$ using the ${ }^{93} \mathrm{Nb} \mathrm{NMR}$ probe [5] also yielded a sharper resonance line and an absence of angular dependence of its width, which is a characteristic of an axially symmetric EFG. This is in contrast with the congruent case, where the resonance is broad and a strong angular dependence of the width is observed.

\section{Conclusions}

Ion beam analysis and hyperfine interaction techniques were used to characterize for the first time near-stoichiometric $\mathrm{LiNbO}_{3}$ crystals. Both techniques show that the lattice of the stoichiometric sample is undisturbed. A comparative study on congruent crystals reveals that in this case two distinct Li sites exist. Computer simulations of the Ii-NRA and Nb-RBS angular scans for planar channeling rule out the models assuming a substantial fraction of antisite niobium to be responsible for the structural disorder. A cation stacking fault of the ilmenite type occurring randomly with a fraction of $2 \%$ reproduces the lithium sites with the observed relative site occupancies.

\section{Acknowledgments}

This work has been funded by JNICT, Portugal, through CERN projects 982/92, 1021/93 and a PhD grant (J.G.M.). 


\section{References}

[1] S.C. Abrahams and P. Marsh, Acta Crystallogr. B 42 (1986) 61

[2] P. Lerner, C. Legras and J.P. Dumas, J. Cryst. Growth 3/4 (1968) 231

[3] K. Nassau and M.E. Lines, J. Appl. Phys. 41 (1970) 533

[4] H. Donnerberg, S.M. Tomlinson, C.R.A. Catlow and O.F. Schirmer, Phys. Rev. B40 (1989) 11909

[5] G.I. Malovichko, V.G. Grachev, E.P. Kokanyan, O.F. Schirmer, K. Betzler, B. Gather, F. Jermann, S. Klauer, U. Schlarb and M. Wöhlecke, Appl. Phys. A56 (1993) 103

[6] J.C. Soares, Nucl. Instr. and Meth. B 64 (1992) 215

[7] I. Földvari, K. Polgár, R. Voszka, R.N. Balasanyang, Crystal. Res. Tech. 19 (1984) 1659

[8] P. Herzog, K. Freitag, M. Reuschenbach and H. Walitzi, Z. Phys. A294 (1980) 13

[9] J.G. Marques, J.G. Correia, A.A. Melo, M.F. da Silva, J.C. Soares and ISOLDE Collaboration, Nucl. Instr. and Meth. B 99 (1995) 645

[10] A. Kling, D. Kollewe and B.C. Grabmaier, Nucl. Instr. and Meth. B 85 (1994) 490

[11] A. Kling, Nucl. Instr. Meth. B, in press.

[12] B. Hauer, R. Vianden, J.G. Marques, N.P. Barradas, J.G. Correia, A.A. Melo, J.C. Soares, E. Diéguez and F. Agulló-López, Phys. Rev. B 51 (1995) 6208 


\section{Figure Captions}

Fig. 1: NRA spectra ( $\alpha$ particles produced in the ${ }^{7} \mathrm{Li}(\mathrm{p}, \alpha){ }^{4} \mathrm{He}$ reaction) for incident $1.5 \mathrm{MeV} \mathrm{H}^{+}$beam in a random direction and aligned with the $\langle 11 \overline{2} 0\rangle$ axis for (a) congruent $\mathrm{LiNbO}_{3}$, (b) stoichiometric $\mathrm{LiNbO}_{3}$.

Fig. 2: NRA and RBS channeling dips for (a) the (0001) plane and (b) <11 $20>$ axis along the (0001) plane. The solid lines are drawn to guide the eyes.

Fig. 3: Comparison of experimental results and computer simulation for the $\mathrm{Li}-$ NRA (0001) angular scan in the case of stoichiometric lithium niobate.

Fig. 4: Comparison of experimental results and computer simulation for the LiNRA (0001) angular scan assuming different defect models for congruent lithium niobate: a) $5.9 \%$ niobium antisites, b) $1.2 \%$ niobium antisites and c) $2 \%$ ilmenite type stacking faults.

Fig. 5: Time dependent anisotropy of the $151-245 \mathrm{keV}$ e- $\gamma$ cascade of ${ }^{111} \mathrm{Cd}$ for (a) congruent $\mathrm{LiNbO}_{3}$, (b) stoichiometric $\mathrm{LiNbO}_{3}$. The c-axis of the single crystals was in the detectors' plane at $45^{\circ}$ with two detectors. 